\title{
Laser Direct Writing on Copper Nanoparticle Film by LightScribe Technique
}

\author{
Akira Watanabe*, Gang Qin and Jinguang Cai \\ Institute of Multidisciplinary Research for Advanced Materials, \\ Tohoku University, 2-1-1 Katahira, Aoba-ku, Sendai 980-8577, Japan
}

\begin{abstract}
Keywords: laser direct writing, laser sintering, LightScribe technique, copper nanoparticle, copper wiring, copper micropattern
\end{abstract}

\section{Introduction}

Recently, the developments of novel materials and technologies to decrease the environmental impact and energy consumption are expected. Printed electronics based on solution process is one of the candidates for the innovation. The concept is further extended to the flexible and printed electronics, where a flexible polymer substrate is employed to realize light weight, flexible, and wearable electronic devices. The additional advantages of the printed electronics are the possibilities of downsizing of an instrument and the reduction of manufacturing costs. As one of the goals of the printed electronics, on-demand production of an electronics device by a desktop size equipment can be considered. Laser processing is expected to contribute the development in the field because of the special selectivity and high processability. In the flexible and printed electronic devices using a polymer substrate, the low temperature processing is a challenging issue because of the low heat resistivity of a polymer substrate compared with an inorganic rigid substrate. The laser processing enables the local heating at the irradiated area which effectively decreases the heat damage and process time. The development of semiconductor laser enables the downsizing of manufacturing equipment. The fabrication of a flexible microsupercapacitor based on laser scribed graphene has been reported by Kaner et al., which gave a clear image of the on-demand production of cost effective devices by a desktop size equipment [1, 2]. They have developed a technique that uses a
DVD burner to fabricate microscale graphenebased supercapacitors. In the laser scribing process, a consumer-grade LightScribe DVD burner was used to produce micropatterns of reduced oxide graphene by laser direct writing. LightScribe was conceived by Hewlett-Packard Company (HP) and brought to market through the joint design efforts of HP's imaging and optical storage divisions in 2004. LightScribe is a direct-to-disc labeling technology which makes graphical patterns onto the surface of a DVD using a semiconductor laser of DVD burner [3]. Unfortunately, the unique technology has discontinued in 2013. HP, Samsung, LaCie and LiteOn have phased out LightScribe drives. The fabrication of graphene microsupercapacitor by LightScribe technology is attractive and smart method, however, the laser scribing process is a black box because the details of the LightScribe technology and consumer-grade LightScribe DVD burners have not been so disclosed. In the previous papers, we have reported the sintering of metal nanoparticle films and the micropatterning by laser direct writing using laboratory laser sources and beam scanners [4-11]. In this paper, we report the formation of copper $\mathrm{Cu}$ micropatterns on a $\mathrm{Cu}$ nanoparticle-coated polymer film by LightScribe technique and discuss the features of the laser scribing process.

\section{Method}

The $\mathrm{Cu}$ nanoparticle ink (Cu1T, $33 \mathrm{wt} \%$ toluene solution) was purchased from ULVAC. A poly(ethylene terephthalate) (PET) film was glued to the surface of a LightScribe DVD disc and then 
the surface was coated by $\mathrm{Cu}$ nanoparticle ink. Laser direct writing on the $\mathrm{Cu}$ nanoparticle-coated PET film was carried out using a LightScribe DVD burner and software (LightScribe system software and simple labeler) [12]. Several LightScribe DVD drives (HP Model, DH-16A6L-CT2, Samsung Model TS-653, Hitachi-LG Model GBC-H20L, GH-40L, LaCie 2d, 300784J) were examined to improve the patterning quality. The emission spectrum from the semiconductor laser of an optical pickup unit was observed by a fiber coupled TE cooled linear CCD array spectrometer (B\&W TEK, BTC112E). The pulse profile of the laser emission was recorded on a digital oscilloscope (Tektronix, TBS 1052B). The laser power was measured by a power meter (OPHIR, NOVA) with a photodiode head (OPHIR, PD200-UV-SH).

\section{Results and discussion}

The fabrication process of the $\mathrm{Cu}$ micropatterns by LightScribe is shown in Fig. $1 . \mathrm{A} \mathrm{Cu}$ nanoparticle-coated PET film attached on a DVD disc is inserted into a LightScribe drive upside-down. In the laser scribing process, the laser beam from an optical pickup unit is focused on the surface of a DVD disc through the pickup lens.

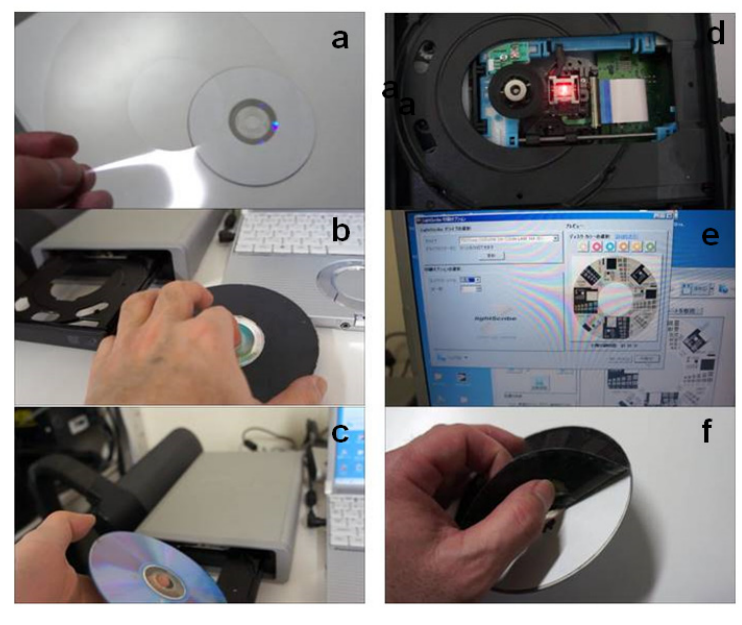

Fig. 1. Fabrication process of $\mathrm{Cu}$ micropatterns by LightScribe. (a) PET film glued to the surface of a LightScribe DVD disc, (b) $\mathrm{Cu}$ nanoparticle-coated disc, (c) insertion of the disc into a LightScribe drive, (d) inside of a LightScribe DVD drive, (e) transfer patterns on the LightScribe software, (f) peeling off patterned PET film from the DVD disc.

Figure 2 shows the optical pickup unit of a LightScribe drive (HP DH-16A6L-CT2). The power of the semiconductor laser in the pickup unit can be controlled by the adjusting screws located at the lower-left in Fig. 2. The laser emissions from the pickup lens measured by a CCD array spectrometer showed two emission lines at around 660 and $780 \mathrm{~nm}$. In the paper reported by HP researchers, the laser source was a $780 \mathrm{~nm}$ laser and the resolution was 600 dots per inch (dpi). They have also reported that LightScribe drives were modified versions of conventional DVD writers as follows: (1) A laser spot of appropriate intensity and size must be created, (2) the spot must be focused on the active layer of the disc, (3) the laser must be pulsed according to the desired data, and (4) the spot must be addressed to the desired location on the surface of the disc [3]. The problem (4) was solved by the speed control provided through the addition of a special encoder in the drive. The detailed mechanism has been reported in the application note of the low speed implementation for LightScribe [13]. Accurate speed control is essential for pixel placement along a track as the disc rotates. A new speed sensor for $40 \mathrm{rpm}$ speed control was developed by Fairchild Semiconductor Corporation. The laser scribing process needs about $25 \mathrm{~min}$ to complete at the highest quality setting because the rotation speed of the disc in LightScribe mode is rather slow.

The Optical micrographs of laser scribed DVD disc by a consumer-grade LightScribe DVD driver (HP Model, DH-16A6L-CT2) is shown in Fig. 3. The laser irradiated area showed the melting of dye layer and the color change to dark brown. Judging from the width of the scribed line, the spot size of the laser beam is approximately $12 \mu \mathrm{m}$. The track density can be estimated to be around 1200 tpi (track per inch) from $20 \mu \mathrm{m}$ track pitch. The track pitch is larger than those of other recording media: $1.6 \mu \mathrm{m}$ for CD-R using a using a $780 \mathrm{~nm}$ laser diode, $0.74 \mu \mathrm{m}$ for DVD-R using a $650 \mathrm{~nm}$ laser

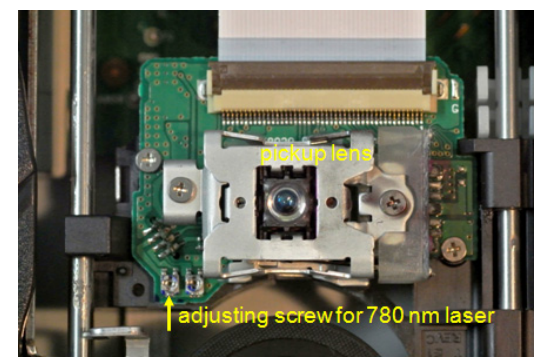

Fig. 2. Optical pickup unit of a LightScribe DVD drive.

diode, and $0.32 \mu \mathrm{m}$ BD-R using a $405 \mathrm{~nm}$ laser diode. The larger track pitch of LightScribe may be 
employed to decrease the scribing time under a slow rotation speed $(40 \mathrm{rpm})$. Kaner et al. have reported the formation of micropatterns of laser scribed graphene with a spatial resolution of $\sim 20$ $\mu \mathrm{m}$, where the maximum power output from a LightScribe DVD drive was $5 \mathrm{~mW}$ [2].

As another labeling technology, Labelflash was introduced by Yamaha and FujiFilm in 2005 [14]. The unique technology burns the image $0.6 \mathrm{~mm}$ deep into the media in as little as $5 \mathrm{~min}$. Figure 4 shows the optical micrographs of a micropattern on the Labelflash DVD disc scribed by a consumergrade Labelflash DVD drive (Sony NEC Optiarc Inc. Model AD-7173S). Remarkable features of Labelflash technology are characterized by the higher resolution and speed of laser scribing. In Fig. 4, the track pitch is approximately $0.70 \mu \mathrm{m}$, which is similar with that of DVD-R using a 650 $\mathrm{nm}$ laser diode. However, a discontinuous dot matrix pattern was observed in a narrow line, which may be due to the laser scribing by pulse irradiation during the high speed spinning. The contrast of the micropattern by LightScribe was higher than that of Labelflash as a result of our test. It may be the reason that LightScribe technology employed $40 \mathrm{rpm}$ speed control to address the laser beam on the desired location on the surface of the disc. We have examined the laser scribing on the graphene oxide coated-Labelflash disc, however, the formation of reduced graphene oxide pattern has not been observed yet. The laser scribing is
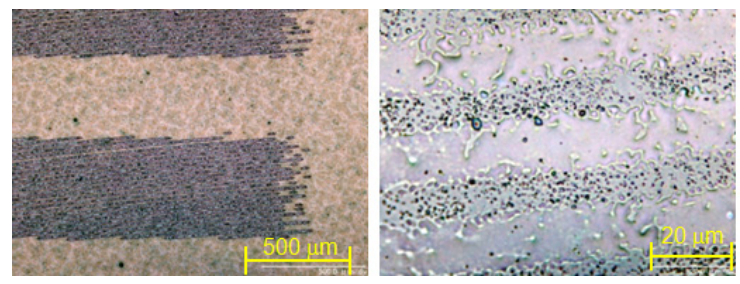

Fig. 3. Optical micrographs of laser scribed DVD disc by a LightScribe DVD drive.
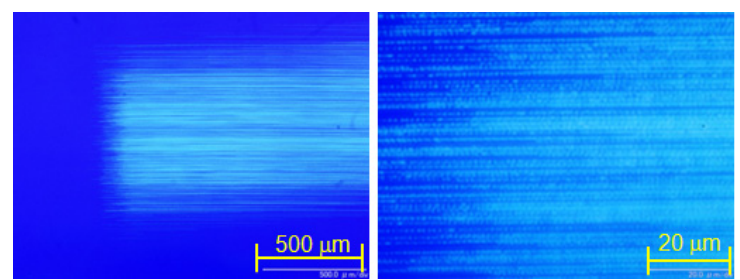

Fig. 4. Optical micrographs of laser scribed DVD disc by a Labelflash DVD drive. influenced by differences in the focal point of laser beam and the disc rotation speed between LightScribe and Labelflash.

The pulse trains during laser scribing process using a consumer-grade LightScribe DVD driver (HP Model, DH-16A6L-CT2) is shown in Fig. 5. The shortest pulse width was around $40 \mathrm{~ms}$ during laser scribing of a 1951 USAF resolution test chart. The linear velocity of the laser beam scan by a LightScribe DVD driver is the range from 25 to $140 \mathrm{~cm} / \mathrm{s}$. These data also suggest the resolution of a few ten of micrometer. During the laser scribing of an entirely-black image, the laser output was almost continuous wave accompanying $76 \mathrm{~Hz}$ modulation as shown in Fig. 5 b.

We applied the LightScribe technique to the laser direct writing on a $\mathrm{Cu}$ nanoparticle-coated PET film with the procedure as shown in Fig. 1. The laser scribing of a 1951 USAF resolution test chart on the film gave a discontinuous patterns consisting of the $\mathrm{Cu}$ lines of ca. $15 \mu \mathrm{m}$ width and the gap of several micrometers as shown in Fig. 6.

The laser power from the pickup lens was increased to decrease the gap between laser scribed lines by adjusting the screw as shown in Fig. 2 . The laser power was controlled by monitoring the
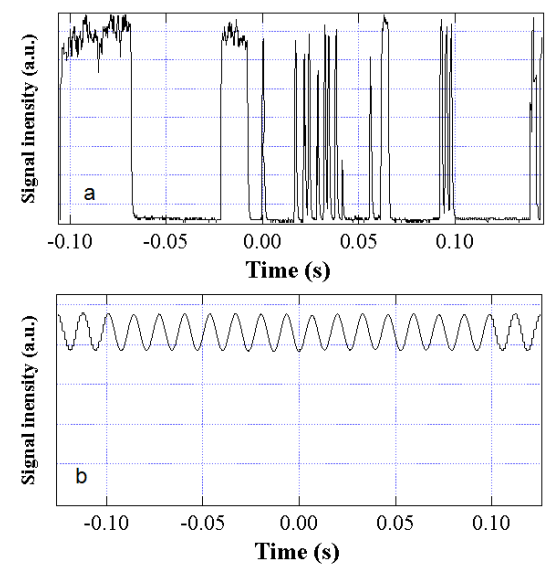

Fig. 5. Pulse trains during laser scribing of (a) resolution test chart and (b) entirely-black image.

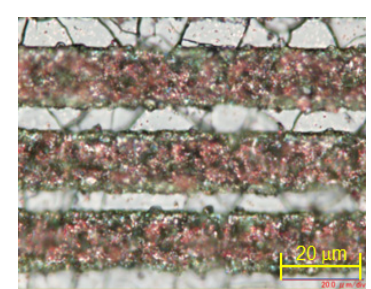

Fig. 6. Optical micrographs of laser scribed $\mathrm{Cu}$ nanoparticle-coated PET film by a LightScrive DVD drive. 


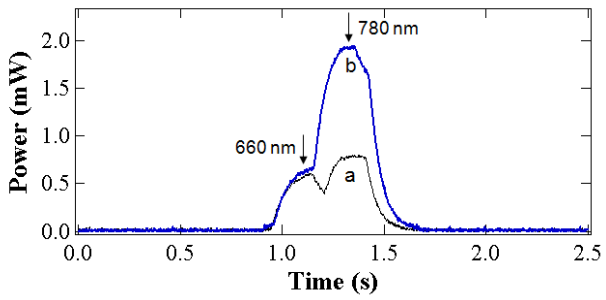

Fig. 7. Laser pulse profile from (a) original and (b) modified DVD drive.

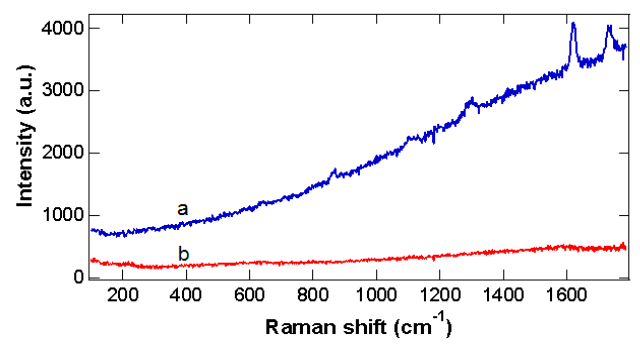

Fig. 9. Raman spectra of $\mathrm{Cu}$ micropattern by LightScribe technique. (a) Intermediate region between scribed lines, (b) laser scribed region.

oscilloscope trace of the laser pulse at the startup of a DVD drive (DH-16A6L-CT2) as shown in Fig. 7. The power of the laser pulse was increased more than twice as much as the original one. The adjustment increased the laser power during LightScribe process from 4.05 to $7.52 \mathrm{~mW}$. Figure 8 shows the optical micrographs of laser scribed $\mathrm{Cu}$ nanoparticle-coated PET film after increasing the laser power. The decrease of the gap between laser scribed lines was clearly shown in Fig. 8b. However, the micro-Raman spectrum showed sharp bands assigned to organic moieties in the intermediate region between the lines (Fig. 9a) whereas the laser scribed region showed no band assigned to organic moieties and oxides of copper (Fig. 9b).

In summary, LightScribe technology has a great possibility to realize an on-demand production by a potable and personal fabrication system. The development of an optimized LightScribe system toward flexible and printed electronics is expected because a consumer-grade one has the limitation in controlling the laser processing conditions and the application.

\section{Acknowledgments}

This work was partially supported by JSPS KAKENHI Grant Number 24360301. This work was also partially supported by MEXT KAKENHI Grant Number 24102004.
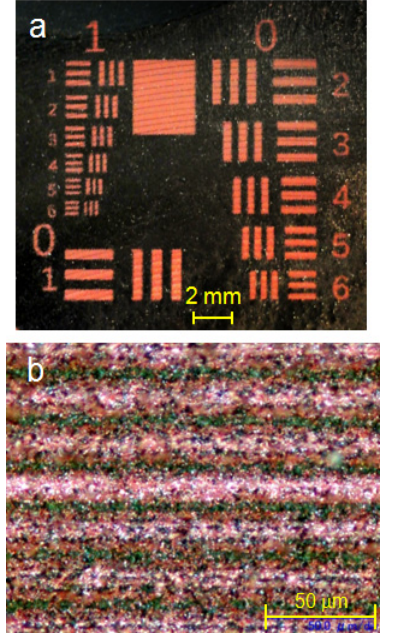

Fig. 8. Optical micrographs of laser scribed $\mathrm{Cu}$ nanoparticle-coated PET film after increasing the laser power. (a) Laser scribed 1951 USAF resolution test chart, (b) enlarged view of consecutive line drawing region.

\section{References}

1. Y V. Strong, C. Dubin, M. F. El-Kady, A. Lech, Y. Wang, B. H. Weiller, R. B. Kaner, ACS Nano, 6 (2012) 1395.

2. M. F. El-Kady and R. B. Kaner, Nat. Commun. 4 (2013) 1475.

3. D. G. Stinson and M. Maguire, LightScribe Direct Disc Labeling, International Symposium on Optical Memory and Optical Data Storage (Optics Society of America, Hawaii, 2005).

4. A. Watanabe, Y. Kobayashi, M. Konno, S. Yamada, T. Miwa, Jpn J. Appl. Phys., 44, (2005) L740.

5. A. Watanabe, Y. Kobayashi, M. Konno, S. Yamada and T. Miwa T., Mol. Cryst. Liq. Cryst., 464 (2007) 161.

6. A. Watanabe and T. Miyashita, J. Photopolym. Sci. Technol., 20 (2007) 115.

7. M. Aminuzzaman, A. Watanabe and T. Miyashita, J. Mater. Chem., 18 (2008) 5092.

8. M. Aminuzzaman, A. Watanabe and T. Miyashita, J. Nanopart. Res., 12 (2010) 931.

9. G. Qin, A. Watanabe, H. Tsukamoto and T. Yonezawa, Jpn. J. Appl. Phys., 53 (2014) 096501-1.

10. G. Qin, G. and A. Watanabe, J. Nanoparticle. Res., 16 (2014) 2684.

11. A. Watanabe, Proc. SPIE 9351 (2015) doi:

$10.1117 / 12.2078081$.

12. LightScribeLabeling.com,

$<$ http://lightscribelabel- ing.com/> (2015).

13. Fairchild Semiconductor Corp., Application Note AN4144, <http://tec.icbuy.com/ uploads/2010/8/9/AN -4144.pdf $>$ (2015)

14. H. Kubo, M. Shibata, S. Yamada, H. Itaga and T. Fushiki, Jpn. J. Appl. Phys. 46 (2007) 3926. 